\title{
A STONE-ČECH COMPACTIFICATION FOR LIMIT SPACES
}

\author{
G. D. RICHARDSON
}

Abstract. O. Wyler [Notices Amer. Math. Soc. 15 (1968), 169. Abstract \#653-306.] has given a Stone-Čech compactification for limit spaces. However, his is not necessarily an embedding.

Here, it is shown that any Hausdorff limit space $(X, \tau)$ can be embedded as a dense subspace of a compact, Hausdorff, limit space $\left(X_{1}, \tau_{1}\right)$ with the following property: any continuous function from $(X, \tau)$ into a compact, Hausdorff, regular limit space can be uniquely extended to a continuous function on $\left(X_{1}, \tau_{1}\right)$.

1. Introduction. The notation and definitions used here can be found in [1] and [2].

Wyler [3] has shown that for every limit space $X$, there is a continuous mapping $j: X \rightarrow B X$, where $B X$ is regular, Hausdorff, and compact, with the following property: for every continuous mapping $f: X \rightarrow Y$, with $Y$ regular, Hausdorff, and compact, there is a unique continuous mapping $g: B X \rightarrow Y$ such that $f=g \circ j$.

We obtain a somewhat similar result with $j$ being an isomorphism.

2. Compactification. Let $(X, \tau)$ be a Hausdorff limit space and $\dot{x}$ the fixed ultrafilter containing $\{x\}$. Denote by $X_{1}=\{\dot{x} \mid x \in X\} \cup$ $\{\vartheta \mid \vartheta$ is a nonconvergent ultrafilter on $X\}$ and $\ddot{x}$ denotes the fixed ultrafilter on $X_{1}$ containing $\{\dot{x}\}$. Let $\mathcal{F}$ be a filter on $X$ and denote by $\hat{F}$ the filter on $X_{1}$ whose base is $\{\hat{F} \mid F \in \hat{F}\}$, where $\hat{F}=\left\{\mathfrak{F} \in X_{1} \mid F \in \mathcal{H}\right\}$.

Define $\tau_{1}(\dot{x})=\left\{\mathfrak{H C} \in X_{1} \mid \mathfrak{H C} \geqq \hat{F}\right.$ for some $\left.\mathfrak{F} \in \tau(x)\right\}$, where $x \in X$, and $\tau_{1}(\mathcal{V})=\left\{\mathfrak{H} \in X_{1} \mid \mathfrak{H} \geqq \hat{\mathcal{V}}\right\}$ for $\mathcal{V}$ a nonconvergent ultrafilter on $X$. From [1], $\hat{\mathfrak{F}} \wedge \hat{\mathcal{G}}=(\mathcal{F} \wedge \mathcal{G})^{\wedge}$. Also $\ddot{x} \geqq \dot{x}^{\wedge}, \dot{\mathcal{V}} \geqq \hat{\mathcal{V}}$, and we easily see that $\tau_{1}$ is a limit structure for $X_{1}$.

Our first goal is to show that $\left(X_{1}, \tau_{1}\right)$ is a compactification of $(X, \tau)$. That is, $(X, \tau)$ is embedded as a dense subspace of $\left(X_{1}, \tau_{1}\right)$.

Lemma 1. The space $\left(X_{1}, \tau_{1}\right)$ is Hausdorff.

Proof. Let $\digamma_{1}, F_{2}$ be any two filters on $X$ and $\mathfrak{F}^{*}$ a filter on $X_{1}$ with $\mathfrak{F}^{*} \geqq \hat{\mathfrak{F}}_{i}(i=1,2)$. It suffices to show that $\mathfrak{F}_{1}$ and $\mathfrak{F}_{2}$ are not disjoint. Let $F_{i} \in \mathscr{F}_{i}(i=1,2)$, then there exists an $F^{*} \in \mathcal{F}^{*}$ such that $F^{*} \subset \hat{F}_{1}$ $\cap \hat{F}_{2}=\left(F_{1} \cap F_{2}\right)^{\wedge}$. Hence $F_{1} \cap F_{2} \neq \varnothing$ and thus $\mathcal{F}_{1}$ and $\mathscr{F}_{2}$ are not disjoint filters.

Received by the editors September 9, 1969.

AMS Subject Classifications. Primary 5422, 5410; Secondary 5453.

Key Words and Phrases. Stone-Čech compactification, limit spaces, ultrafilters. 
Lemma 2. The space $\left(X_{1}, \tau_{1}\right)$ is compact.

Proof. Let $\mathcal{V}^{*}$ be an ultrafilter on $X_{1}$. Define $\mathcal{F}=\left\{F \subset X \mid \hat{F} \in \mathcal{V}^{*}\right\}$. Clearly $\mathcal{F}$ is a filter on $X$. Moreover, if $A \cup B \in \mathcal{F}$ then $(A \cup B)^{\wedge}$ $=\hat{A} \cup \hat{B} \in \mathcal{V}^{*}$ and hence from [1] either $\hat{A}$ or $\hat{B}$ belong to $\mathcal{V}^{*}$. Therefore, either $A$ or $B$ belong to $\mathcal{F}$ and again from [1] we have that $\mathcal{F}$ is an ultrafilter on $X$.

Clearly $\mathcal{U}^{*} \geqq \hat{\mathfrak{F}}$. If $\mathfrak{F} \in \tau(x)$ for some $x \in X$, then we have that $V^{*} \in \tau_{1}(\dot{x})$. On the other hand, if $\mathscr{F}$ does not converge on $X$ then $\mathfrak{F} \in X_{1}$ and thus $\mathcal{V}^{*} \in \tau_{1}(\mathfrak{F})$. Hence $\left(X_{1}, \tau_{1}\right)$ is compact.

Define $i: X \rightarrow X_{1}$ such that $i(x)=\dot{x}$. Since the restriction of the filter $\hat{F}$ to $i(X)$ is $i(\mathfrak{F})$, we have that $i$ is an isomorphism onto $i(X)$.

Let $v \in X_{1}$. Then $i(v) \geqq \hat{V}$ and it follows that $i(X)$ is dense in $X_{1}$. Therefore, $\left(X_{1}, \tau_{1}\right)$ is a compactification of $(X, \tau)$.

Theorem. Let $f:(X, \tau) \rightarrow\left(X_{2}, \tau_{2}\right)$ be continuous with $\left(X_{2}, \tau_{2}\right)$ compact, regular, and both spaces Hausdorff. Then $\left(X_{1}, \tau_{1}\right)$ is a compactification of $(X, \tau)$ such that $f$ has a unique continuous extension to $X_{1}$.

Proof. Only the latter part remains to be proved. Define $f_{1}: X_{1} \rightarrow X_{2}$ such that $f_{1}(v)=\lim f(\mathcal{V})$ for $V \in X_{1}$. From [1], and $[2], f_{1}$ is well defined. In order to prove that $f_{1}$ is continuous, it suffices to show that for $\mathfrak{F}^{*} \geqq \hat{\mathfrak{F}}, \mathcal{F}$ is a filter on $X$, then $f_{1}\left(\mathfrak{F}^{*}\right) \geqq \operatorname{cl}(f(\mathcal{F}))$. Let $F \in \mathcal{F}$. Then there is an $F^{*} \in \mathcal{F}^{*}$ such that $F^{*} \subset \hat{F}$. We claim that $f_{1}\left(F^{*}\right) \subset \operatorname{cl}(f(F))$. If $V \in F^{*}$, then $F \in \mathcal{V}$ and from [2], $f_{1}(\mathcal{V}) \in \operatorname{cl}(f(F))$ and thus our claim follows. Hence $f_{1}$ is continuous.

Clearly $f_{1}$ is the unique continuous extension of $f$ to $X_{1}$.

\section{REFERENCES}

1. N. Bourbaki, General topology. Part I, Hermann, Paris and Addision-Wesley, Reading, Mass., 1966. MR 34 \#5044a.

2. H. R. Fischer, Limesräume, Math. Ann. 137 (1959), 269-303. MR22 \#225.

3. O. Wyler, The Stone-Cech compactification for limit spaces, Notices Amer. Math. Soc. 15 (1968), 169. Abstract \#653-306.

East Carolina University, Greenville, North Carolina 27834 and North Carolina State University, Raleigh, North Carolina 27607 